La Revue

des Droits

de l'Homme

\section{La Revue des droits de l'homme}

Revue du Centre de recherches et d'études sur les droits fondamentaux

9 | 2016

Revue des droits de l'homme - $\mathrm{N}^{\circ} 9$

\title{
Le principe de légalité de la peine en droit pénal international, points de force et de faiblesse
}

\section{Maria Stefania Cataleta}

\section{OpenEdition}

\section{Journals}

Édition électronique

URL : http://journals.openedition.org/revdh/1868

DOI : $10.4000 /$ revdh. 1868

ISSN : 2264-119X

\section{Éditeur}

Centre de recherches et d'études sur les droits fondamentaux

\section{Référence électronique}

Maria Stefania Cataleta, « Le principe de légalité de la peine en droit pénal international, points de force et de faiblesse », La Revue des droits de I'homme [En ligne], 9 | 2016, mis en ligne le 03 mars 2016, consulté le 09 octobre 2020. URL : http://journals.openedition.org/revdh/1868; DOI : https://doi.org/ 10.4000/revdh.1868

Ce document a été généré automatiquement le 9 octobre 2020.

Tous droits réservés 


\title{
Le principe de légalité de la peine en droit pénal international, points de force et de faiblesse
}

\author{
Maria Stefania Cataleta
}

\section{Introduction}

Dans le cadre de la justice pénale internationale, le régime de la sanction concerne une partie des règles relatives au procès équitable où le droit de l'accusé à une peine juste, humaine, visant à sa réinsertion sociale, déjà connue ou au moins prévisible avant la perpétration de la conduite et enfin prononcée suite à une procédure équitable, doit se concilier avec un aspect plus symbolique, propre à ce type de justice, à savoir l'intérêt de la communauté internationale à une condamnation sévère pour des crimes particulièrement odieux tels que les crimes de droit international. Pour cette raison, la légalité de la peine est abordée différemment dans le contexte national et dans le contexte international, où la pratique des Tribunaux pénaux internationaux (T.P.I.). a certainement influencé la réglementation de la Cour pénale internationale (C.P.I.). La présente lecture du système répressif international vise à analyser le principe de légalité de la peine dans le cadre du procès pénal international et en souligner les points de force et de faiblesse à la lumière de la pratique juridictionnelle internationale. En particulier, cette contribution se propose d'examiner au vu du caractère incertain du principe de légalité de la peine en droit pénal international (I) comment sont interprétés et appliqués les principes de proportionnalité et d'individualisation de celle-ci (II).

\section{Un principe incertain}

2 Le 13 novembre 2015, les juges de la Chambre d'appel de la Cour pénale internationale ont réduit la peine d'emprisonnement à M. Katanga, qui, le 23 mai 2014, avait été 
condamné à 12 ans d'emprisonnement pour les crimes contre l'humanité et crimes de guerre commis dans le village de Bogoro, en République démocratique du Congo (R.D.C.). Par cette décision les victimes et les autorités congolaises craignent l'effet déstabilisateur du retour de M. Katanga en R.D.C., car la peine prendra fin le 18 janvier 2016. Au total, il aura purgé 9 ans et 4 mois $^{1}$. Conformément au principe de légalité de la peine, tout système juridique doit faire en sorte qu'avant la perpétration d'une infraction pénale un individu connaisse, doive pouvoir connaître, prévoir ou au minimum être mis en condition de savoir qu'une conduite donnée est contraire à la loi et, qu'en tant que telle, elle est passible de sanction pénale. En droit international pénal (D.I.P.), ce qui est désormais exigé pour la définition des crimes n'est pas encore vraiment exigé en ce qui concerne les peines, un domaine où une large liberté domine encore parmi les juges. Dans cette perspective, l'analyse du principe de la légalité de la peine dans le milieu pénal international doit tenir compte de la particularité de ce système répressif, qui n'a pas de tradition judiciaire uniforme à cet égard. A l'exception des Tribunaux de Nuremberg et de Tokyo, le seul élément commun au sein de la justice pénale internationale est l'interdiction absolue de la peine de mort, interdite en droit international ${ }^{2}$. L'opposition de plusieurs pays, notamment les États européens et latinoaméricains, a porté à l'exclusion de la peine capitale du Statut de la Cour pénale internationale. Malgré cela, son Statut n'a pas été en mesure de codifier l'interdiction en tant que norme du droit international ${ }^{3}$. En ce qui concerne les peines appliquées par les juridictions pénales internationales, il manque une véritable pratique consolidée. En général, la création des juridictions internationales ou internationalisées, et par conséquent la création des normes pour leur fonctionnement, y compris la réglementation sur la peine, a souvent suivi la perpétration des crimes. À l'exception de l'Accord de Rome, les instruments internationaux de répression des violations du droit international humanitaire et des crimes qui menacent la paix ont toujours été peu satisfaisants dans l'indication des peines, en confiant aux États leur exécution. Dans le meilleur des cas, les traités et les déclarations internationales prescrivent aux États de prendre toute mesure nécessaire pour appliquer une sanction juste et appropriée $e^{4}$. À cet égard, par rapport à toute conduite criminelle sanctionnée, les systèmes juridiques nationaux établissent une norme qui en général contient aussi bien la description de la conduite que l'indication de la sanction à appliquer au cas d'espèce ${ }^{5}$. En revanche, dans le milieu international, en raison des divergences dans l'appréciation de la gravité des crimes internationaux de la part de systèmes juridiques différents, mais surtout à cause de l'existence d'un éventail trop différencié de sanctions pénales au sein des différentes traditions juridiques, il a été très difficile de fixer une grille de peines maximales ou minimales pour chaque crime international. Dans le cadre de la justice pénale internationale, l'indication stricte des sanctions concerne simplement l'énumération des types de peines applicables, à savoir l'emprisonnement et les sanctions pécuniaires accessoires. L'incertitude qui a caractérisé pendant longtemps la définition stricte et détaillée des éléments des crimes de droit coutumier s'est donc reflétée sur la sanction. Cela est démontré par le fait que, dans le choix de la peine applicable, les instances pénales internationales ont commencé par une application extrême de la peine de mort, admise par les Tribunaux de Nuremberg et de Tokyo, et ont conclu jusqu'à l'application de peines accessoires pécuniaires, telles que l'amende et la confiscation ${ }^{6}$, ou de toute mesure visant à la réparation en faveur des victimes, sanction propre à la C.P.I. ${ }^{7}$. 
3 Le Statut de Rome, à l'exception d'une indication des types de peines applicables et des critères pour leur détermination - à savoir la gravité du crime, la situation personnelle du condamné ${ }^{8}$, les conclusions et les preuves pertinentes présentées au procès9-, présente une imprécision en ce qui concerne le quantum, ce qui donne au juge international une marge d'arbitraire plutôt large en matière de peines. De plus, le fait que, dans la détermination du quantum de la peine, le juge fasse référence à la gravité du crime et surtout à la situation personnelle du condamné peut provoquer des distorsions et des disparités à l'égard des accusés condamnés pour les mêmes crimes. Il peut donc bien se produire qu'une Chambre estime de manière différente des facteurs identiques ou des situations analogues et inflige à des condamnés divers un nombre d'années différent pour les mêmes crimes, en générant ainsi des inégalités ${ }^{10}$. Afin de déterminer la peine, les Statuts des T.P.I. ont permis aux juges de faire référence aux dispositions propres au système national, une pratique qui a été ensuite confirmée par la jurisprudence ${ }^{11}$. La référence à la loi nationale et à la pratique des tribunaux nationaux a donc certainement fourni un outil important pour combler la règlementation lacunaire des T.P.I. en ce qui concerne une liste de sanctions applicables et les critères aptes à calibrer la peine à infliger. Toutefois, loin de faciliter le travail des T.P.I., la référence aux peines admises dans les systèmes nationaux n'a pas évité des problèmes, en raison du fait que, d'une part, les systèmes nationaux ne prévoyaient pas les mêmes crimes internationaux de compétence que les T.P.I., et d'autre part, parmi les peines prévues au niveau national, la peine de mort pouvait être admise. De plus, en ce qui concerne le premier aspect, la non-prévision des crimes internationaux dans les systèmes nationaux a permis aux Tribunaux ad hoc d'appliquer les peines établies pour certains crimes nationaux présentant une gravité jugée équivalente à celle des crimes internationaux, ce qui implique l'application du critère de l'analogie, interdit par le principe de légalité ${ }^{12}$. Dans l'affaire Erdemović, le T.P.I.Y. a argué que les crimes contre l'humanité, une espèce juridique absente dans le Code Pénal de l'ex-Yougoslavie, devaient être considérés équivalents à d'autres crimes prévus dans le système national présentant la même nature et gravité que les crimes contre l'humanité13 ; cela permettait la détermination d'une peine importante, conformément à l'orientation du Conseil de Sécurité qui demandait des peines sévères pour ces crimes si graves. Toutefois, une telle interprétation fait fi du le principe de légalité de la peine, considéré donc comme un principe non absolu, même si la référence à la législation nationale avait "pour vocation d'apaiser la défense qui pourrait avoir la tentation de faire valoir le principe nulla pona sine lege ${ }^{14}$. L'expérience des juridictions précédentes à la C.P.I. a donc montré une focalisation sur les aspects quantitatifs plutôt que qualitatifs de la peine en droit international pénal.

4 Le Statut de la Cour donne une formulation du principe de légalité de la peine qui se détache de la formulation classique généralement utilisée dans les systèmes pénaux nationaux, selon lesquels le juge ne peut appliquer ni une peine qui n'était pas prévue au moment de la perpétration du crime, ni une peine plus lourde que celle prévue au moment de la perpétration du crime. En effet, selon la formulation de l'art. 23 du Statut de Rome, la seule limite que les juges de la Cour rencontrent au moment de la détermination et de l'application de la peine est sa conformité au Statut ${ }^{15}$. Or, le critère de la conformité au Statut se détache de la réglementation des T.P.I., où la référence à la pratique générale adoptée par les instances nationales de l'ex-Yougoslavie et du Rwanda rendait incertaine le principe de sécurité de la peine, les juges n'étant pas obligés à la respecter, puisque cette pratique était simplement un support ${ }^{16}$. Déjà en 
1994, dans le Projet de Statut que la Commission de droit international (C.D.I.) avait soumis à l'A.G., la référence au principe de légalité de la peine avait été plutôt générique, comprenant les peines de l'incarcération jusqu'à perpétuité et de la sanction pécuniaire. Il était également indiqué que la Cour devait prendre en considération le droit appliqué par les États, en particulier l'État de l'accusé ${ }^{17}$. Ensuite, l'obligation de conformité aux systèmes nationaux fut éliminée du fait d'un manque d'accord entre les États sur ce point ${ }^{18}$. Plusieurs pays demandaient des règles de procédure établissant avec précision la peine maxima et minima relative à l'emprisonnement, ainsi qu'une détermination précise des circonstances aggravantes et atténuantes et des critères pour le cumul des peines en cas de crimes multiples ${ }^{19}$. En revanche, l'interdiction du recours à l'analogie fut établie ${ }^{20}$. Enfin, le principe de légalité de la peine fut introduit dans l'article 23 du Chapitre III, consacré aux principes généraux du droit pénal ${ }^{21}$. Résultat d'un long travail d'élaboration, l'article 23 du Statut impose le respect du principe de légalité de la peine, même s'il manque une grille précise concernant les peines d'emprisonnement. À cet égard, il faut noter que l'article 77 indique en tant que peines applicables l'emprisonnement jusqu'à 30 ans au plus et celui à perpétuité, auxquelles il faut ajouter l'amende et la confiscation. La garantie contre tout risque d'arbitraire au moment de la sanction est donc assurée uniquement par le Statut qui admet les sanctions qui se conforment à ses dispositions. De façon concrète, il s'agit d'une limite qui s'impose aux juges sur toute décision en matière de preuve, puisque la durée de la peine dépend de la gravité de la conduite et de la personnalité du condamné, un critère permettant de calibrer avec plus de précision la sanction sur la base de sa fonction réelle de rééducation. En ce qui concerne la peine d'emprisonnement, puisque la limite maximale est indiquée, les juges jouissent d'un pouvoir discrétionnaire considérable dans la détermination du nombre d'années approprié à chaque conduite. Par conséquent, l'ampleur de la marge d'appréciation du quantum peut conduire à des prononciations très différentes pour les mêmes crimes par rapport à d'autres juridictions internationales et parfois par rapport à d'autres chambres de la même juridiction. Le critère général de la "conformité » au Statut limite donc le pouvoir de la Cour, mais également celui des États où les personnes condamnées par la Cour purgent leur peine de détention, auxquels il n'est pas permis d'infliger des peines autres que celles décidées par la Cour. À cet égard, il faut souligner que l'art. 78 limite les juges dans leur pouvoir discrétionnaire dans la mesure où il fait référence à la gravité du crime et à la situation personnelle du condamné. Tout autre aspect lié à la graduation de la peine, qui est abordé par l'alinéa 1 de l'article mentionné, est réglé plus spécifiquement par le Règlement de procédure et de preuve (R.P.P.), qui prévoit les conditions de l'emprisonnement ou les modalités pour exécuter les peines pécuniaires ${ }^{22}$. Le R.P.P. indique également les critères pour considérer la peine déjà purgée avant la fin du procès, afin de déterminer la peine finale et aborde en général toute question concernant le moment de l'exécution de la peine ${ }^{23}$, point central pour son efficacité.

5 Dans le cadre juridique international, la fonction propre à la peine est plus complexe que dans le milieu national, puisqu'elle doit neutraliser et punir le coupable, le dissuader de commettre d'autres crimes, permettre sa réinsertion sociale et, par le biais de la réprobation collective, favoriser la réconciliation, la paix et l'ordre international ${ }^{24}$. En effet, dans le cadre d'un procès pénal international, caractérisé par ses propres règles et finalités, il peut se présenter la tentation d'affirmer que l'intérêt de la justice et de la collectivité prévaut sur l'intérêt de l'accusé à faire valoir ses droits, 
mais surtout à avoir l'occasion d'une deuxième chance. Cependant, si dans le contexte national le respect de la légalité de la peine limite le pouvoir des juges dans la répression pénale, dans le système international, le principe a également la fonction d'éliminer tout doute de pressions politiques provenant de l'extérieur qui pourrait influencer l'activité de la juridiction internationale, d'autant plus que les crimes internationaux prolifèrent dans des contextes politiques particulièrement conflictuels, comme les guerres civiles où il y a toujours des factions politiques plus fortes et influentes. Dans le contexte international, le principe de légalité a la fonction ultérieure d'éviter que la décision sur la peine produise des inégalités liées à l'éducation, à la culture, aux opinions personnelles, religieuses ou politiques différentes de juges provenant de tous les coins du monde. Par ailleurs, une décision peu scrupuleuse sur la peine est toujours sujette à des critiques de la part de l'opinion publique, très attentive à vérifier l'impartialité et l'indépendance de l'instance. En même temps, il est important que la décision évite une application trop stricte du principe de légalité, dans la mesure où la peine doit toujours tenir compte de la gravité du crime et des conditions personnelles de l'auteur ${ }^{25}$. En termes généraux, la doctrine classique indiquait deux approches pour encadrer la peine, l'une prospective et l'autre restitutive. Selon la première, la peine a une fonction de dissuasion pour l'avenir. Pour cela, il est important qu'elle soit connue à l'avance - et de réhabilitation sociale du coupable $^{26}$. La deuxième approche considère la peine en tant que châtiment en raison du mal porté à la société. Il s'agit d'une rétribution qui ne doit cependant pas dépasser la gravité du crime commis et qui, à travers la réparation, vise également à rétablir le statu quo ante.

6 En matière de peine, le Statut de Rome reste générique, en particulier en ce qui concerne l'absence de paramètres à adopter pour la détermination du quantum. En combinaison avec les critères indiqués dans l'art. 76(1) déjà cité, aux termes de l'article 78(1), les indications pour l'individualisation de la peine sont donc la gravité du crime et la situation personnelle du condamné27. L'objectif reste une peine "juste", dans la mesure où elle doit punir avec sévérité mais par le biais d'un châtiment humain, c'està-dire qui préserve la dignité de l'accusé de tout propos vindicatif ${ }^{28}$. L'individualisation de la peine nécessite qu'elle soit proportionnelle à la culpabilité du condamné et tienne compte des circonstances aggravantes et atténuantes, comme sa collaboration ou son plaidoyer de culpabilité29. En d'autres termes, il faut moduler la peine selon les principes d'individualisation et de proportionnalité afin qu'elle ne soit pas porteuse de disparités, et, en même temps, qu'elle ne présente une gravité ni supérieure ni inférieure à la gravité du crime commis. La peine doit donc être juste, c'est-à-dire qu'elle doit s'adresser à une personne déterminée, tout en restant proportionnée à la gravité aussi bien objective que subjective du crime ${ }^{30}$. Mais, l'individualisation de la peine dérive du degré de responsabilité du condamné plutôt que de la gravité du crime. En effet, ce qui ressort est le rôle, la personnalité, l'éducation, l'âge, la propension délictueuse, les antécédents judiciaires, les conditions mentales, physiques, familiales, financières, sociales et le niveau d'instruction du condamné ${ }^{31}$. Par conséquent, une disparité de la peine est admissible si elle est le résultat du processus d'individualisation et non d'une discrétion arbitraire et injustifiée de la part des juges ${ }^{32}$.

Or, la mesure objective de la gravité du crime est donnée par la loi, alors que la mesure subjective du crime découle des circonstances dans lesquelles le crime s'est accompli, pouvant aggraver la peine ou l'atténuer ${ }^{33}$. La nécessité de prévoir tout l'éventail des circonstances possibles dans le R.P.P. de la C.P.I. avait été défendue sans succès pendant 
la Conférence de Rome ${ }^{34}$. Toutefois, plus que les règles de procédure, la jurisprudence des T.P.I. a beaucoup contribué à la détermination des circonstances. Parmi les circonstances aggravantes, il faut considérer l'étendue et la gravité des dommages et des préjudices causés aux victimes et aux membres de leur famille, le nombre, l'âge, le genre de celles-ci et en général, leur vulnérabilité, l'abus de pouvoir, la préméditation, le sadisme, l'emploi de moyens ou de modalités particulièrement cruels et l'inclination criminelle de l'accusé, le mobile discriminatoire, la présence d'autres condamnations pénales antérieures ${ }^{35}$. La jurisprudence des T.P.I. a retenu in re ipsa les circonstances aggravantes quand il s'agit de crimes de génocide et contre l'humanitée ${ }^{36}$. En revanche, les circonstances atténuantes limitent le quantum de la peine, sans cependant réduire la gravité du crime. La coopération avec l'organe de poursuite, surtout si elle est considérée comme sérieuse et étendue, peut réduire la peine. De façon analogue, le fait d'avoir fourni des éléments probants utiles dans d'autres affaires, la reddition volontaire, l'indemnisation des victimes, l'admission de culpabilité, la rémission au tribunal, le plaidoyer de culpabilité, la contrition et les remords désintéressés démontrés envers les victimes en audience publique, sont des facteurs de réduction de la peine dans la perspective d'une réhabilitation du coupable ${ }^{37}$. La jurisprudence de la Cour, dans l'affaire Katanga, a indiqué également le soutien actif de l'accusé au processus de désarmement et de démobilisation des enfants soldats ${ }^{38}$. Par contre, la qualité officielle n'est pas un motif d'exonération de la responsabilité pénale ni de diminution de la peine ${ }^{39}$. L'ordre du supérieur, invoqué en vain devant le Tribunal de Nuremberg ${ }^{40}$, est admis comme motif de diminution de la peine, puisque la désobéissance implique normalement des représailles contre le subordonné ou sa famille ${ }^{41}$. Toutefois, dans ce cas, la diminution de la peine n'est pas automatique mais subordonnée à la preuve de la réprobation, du rôle subalterne dans l'échelle de commande, du degré limité de participation, de l'état de contrainte ou d'extrême nécessité, de la résistance du subordonné par rapport à l'ordre reçu, de l'éventuelle volonté ou activité employée pour réduire les effets dangereux du crime et pour alléger les souffrances à l'encontre des victimes ${ }^{42}$. Toujours dans l'affaire Katanga, la défense a affirmé que la violation des standards internationaux relatifs aux droits de l'accusé peut atténuer la mesure de la peine, ce qui a été retenu par la Cour ${ }^{43}$, le respect des conditions d'exécution de la sanction étant le fondement pour la rééducation du condamné.

\section{Des peines proportionnées et individualisées}

Plusieurs questions concernant l'exécution de la peine, la liberté conditionnelle, les conditions de détention et les mesures alternatives à la détention restent incertaines ${ }^{44}$. Il s'agit d'aspects cruciaux pour garantir l'efficacité de la sanction. Selon l'art. 77, les peines que la Cour peut appliquer sont: l'emprisonnement jusqu'à trente ans et l'emprisonnement à perpétuité pour les cas d'une gravité exceptionnelle. À l'emprisonnement il est possible d'ajouter une amende et la confiscation ${ }^{45}$. Puisque la Cour est compétente à l'égard des seules personnes physiques, la responsabilité pénale des personnes morales n'a pas été retenue au cours des travaux préparatoires. À l'exception des sanctions pécuniaires, l'emprisonnement est donc la seule peine principale appliquée par la C.P.I. ${ }^{46}$. Au cours des travaux de la C.D.I., le Projet de Statut prévoyait l'emprisonnement à vie, malgré les objections contre l'emprisonnement à perpétuité, considéré comme équivalent à la peine capitale ${ }^{47}$. À la différence des Statuts 
des T.P.I., où l'emprisonnement à perpétuité n'était pas indiqué et cependant était infligé par les juges en vertu de leur interprétation statutaire en cas de condamnation pour génocide, le Statut de Rome admet l'emprisonnement d'une durée de 30 ans ou plus et l'emprisonnement à perpétuité ${ }^{48}$. L'application de l'incarcération à vie, répudiée par certaines démocraties dans lesquelles cette peine est considérée comme une pratique de torture, dénature le caractère humain et non dégradant de la peine que le Statut de Rome devrait assurer. Mais, au-delà des critères indiqués, la réglementation du Statut en la matière témoigne de l'évolution du principe de légalité de la peine au niveau international, auparavant caractérisé par une inégalité importante par rapport au contexte national - qui, en général, prévoit pour chaque crime un minimum et un maximum de la peine à infliger -, dans la mesure où l'arbitraire qui caractérisait le moment de la prononciation de la peine de la part du juge international a laissé la place à un pouvoir discrétionnaire plus limité du fait d'une réglementation plus détaillée ${ }^{49}$. Or, l'article 77(1)(b), en matière d'emprisonnement à perpétuité requiert que la gravité du crime soit jugée comme "extrême ", même si l'on parle de crimes déjà très graves par leur caractère. En effet, l'extrême gravité trouve un sens si l'on considère l'intention spécifique de l'accusé et la gravité des dommages causés par le crime. Dans cette perspective, l'extrême gravité sera alors considérée par rapport à l'ampleur des effets produits et certaines conduites, comme celles génocidaires, seront in re ipsa d'une gravité extrême. Dans le cadre des crimes internationaux, une certaine doctrine ainsi qu'une jurisprudence nient toute hiérarchie liée à la gravité des $\operatorname{crimes}^{50}$, en les considérant tous de la même gravité, alors que d'autres placent au sommet le crime d'agression ou le génocide, défini par certains comme le crime des crimes ${ }^{51}$. À côté de l'art. 78(1) déjà énoncé, l'article 77 requiert spécifiquement la prise en compte de la situation personnelle du condamné, comme l'un des critères pour justifier une peine très sévère. Dans ce cas, le paramètre suivra néanmoins une procédure d'évaluation plus commune, où la position de pouvoir de l'accusé au moment du crime et le degré de cruauté employée seront la mesure pour rendre le responsable destinataire d'une peine plus lourde, du fait de l'intention spécifique démontrée dans la perpétration du crime $^{52}$. En l'absence d'élément causal, lié aux effets produits, et d'élément intentionnel, lié à l'état d'âme particulier, la peine maximale ne pourra dépasser les 30 ans. L'emprisonnement à perpétuité doit donc se «justifier " principalement par la gravité extrême du crime et par la situation personnelle du condamnéf3. Enfin, il est important de souligner que, pour justifier l'infliction d'une peine aussi grave que la peine à perpétuité, la démonstration de l'extrême gravité du crime et d'une situation personnelle donnée ne suffisent pas, étant donné qu'il faut que ces deux facteurs soient "attesté[e]s par l'existence d'une ou de plusieurs circonstances aggravantes " ${ }^{54}$. En d'autres termes, l'évaluation de la preuve est plus rigoureuse pour la peine la plus grave, qui nécessite que les deux seuls éléments objectif et subjectif la justifiant soient impérativement corroborés par la présence d'au moins une circonstance aggravante ${ }^{55}$.

9 L'amende et la confiscation sont des peines que la Cour peut ajouter à la peine principale de l'emprisonnement ${ }^{56}$. Il s'agit de peines à caractère civil, affectant le condamné dans sa condition économique et sociale, qui n'étaient pas prévues par les Statuts des T.P.I., lesquels se limitaient à indiquer la restitution comme peine accessoire. Le caractère civil de l'amende et de la confiscation répond à la fonction de la C.P.I. de dissuader et de punir le responsable, d'éviter que celui-ci ne s'enrichisse par le crime, d'assurer une juste réparation aux victimes et d'indemniser symboliquement la société ${ }^{57}$. Des difficultés pratiques existent pour activer la machine de la sanction, qui 
n'est pas automatique : elles concernent la coopération étatique absolument nécessaire pour permettre la localisation et l'identification des biens liés aux crimes ou l'appréciation de la preuve justifiant l'application de peines accessoires. À cet égard, la Cour peut se faire assister par des experts pour prouver l'existence des dommages causés par le crime et pour déterminer leur importance, ce qui peut s'avérer parfois très compliqué ${ }^{58}$. Les critères pour justifier la condamnation à la peine de l'amende sont contenus dans la règle 146 du R.P.P.: dans ce cas, se configure un aspect strictement procédural du principe de légalité de la peine. Après avoir déterminé si l'emprisonnement est une peine suffisante ou non, la Chambre considère les moyens financiers du condamné et dans quelle mesure le profit personnel tiré par l'auteur était un mobile du crime. Les juges évaluent également tout dommage et préjudice causé, en observant que, pour déterminer le montant approprié, celui-ci ne doit pas dépasser les trois quarts de la valeur des biens de la personne condamnée et de tout avoir identifiable, liquide ou réalisable. L'intention est donc de permettre au condamné de continuer à répondre à ses besoins financiers personnels et familiaux, dans l'optique de sa réhabilitation. Si le condamné ne procède pas au paiement de l'amende dans le délai établi, son emprisonnement peut être prolongé, mais seulement après que le Président de la Cour l'a écouté à huis clos, en présence d'un conseil. En tout cas, l'allongement ne pourra pas avoir pour effet de porter la durée totale de la détention à plus de 30 ans, car cela se révèlerait contraire à toute finalité rééducative de la peine. Quant à la confiscation, elle doit concerner seulement les biens, les revenus, les avoirs et les profits qui découlent directement ou indirectement du crime, et que la Cour doit identifier et localiser sans porter préjudice à tout tiers de bonne foi ${ }^{59}$. Puisque la sanction pécuniaire est également destinée à la réparation des victimes ${ }^{60}$, la Cour peut, avant de rendre son ordonnance, inviter les représentants du Fonds au profit des victimes à soumettre leurs observations ${ }^{61}$. En effet, la réglementation pour l'exécution des peines de l'amende et de la confiscation est commune à celle sur les mesures de réparation ${ }^{62}$.

La réglementation sur l'exécution de la peine confirme le lien entre le système national et international, puisque la C.P.I. recourt à la coopération volontaire, à l'assistance et à la disponibilité des États pour mettre en exécution leurs condamnations, du fait du manque d'instruments institutionnels d'exécution ${ }^{63}$. Selon le Statut de Rome "les États Parties doivent partager la responsabilité de l'exécution des peines d'emprisonnement conformément aux principes de répartition équitable énoncés dans le règlement de procédure et de preuve", afin de traduire par la coopération l'esprit conventionnel du Statut ${ }^{64}$. Toutefois les États doivent manifester leur disponibilité, ou du moins, indiquer les limites de leur disponibilité, comme la disponibilité sous réserve d'accepter uniquement un certain nombre de condamnés ou leurs ressortissants et résidents ${ }^{65}$. À travers l'interaction entre dimension nationale et internationale, le système d'exécution de la peine propre à la justice pénale internationale présente, dans le Statut de la C.P.I., un caractère novateur et une réglementation toute particulière ${ }^{66}$. Dans ce domaine, la réglementation de la C.P.I. diverge de celle des T.P.I. à cause de sa compétence subsidiaire par rapport à la primauté des Tribunaux ad hoc sur les juridictions nationales. Ce qui est certain est que la peine infligée a un caractère international, alors que la réglementation régissant son exécution est nationale, bien que la Cour ne perde pas son contrôle contre tout dépassement grave des règles conventionnelles en matière de traitement digne des détenus, règles largement acceptées au niveau international. En général, la Cour maintient son autorité sur la 
purgation de la peine, y compris sa durée ${ }^{67} e t$, après un certain nombre d'années déjà purgées, la Cour procède à un examen de la possibilité d'une réduction de la durée de la peine $^{68}$. En revanche, les États Parties sont obligés d'exécuter la peine, ne pouvant la modifier en aucun $\operatorname{cas}^{69}$, et doivent faire en sorte que les communications entre le condamné et la Cour soient libres et confidentielles ${ }^{70}$. Le contrôle que la Cour exerce quant à la conformité de l'exécution aux principes internationaux sur la protection des détenus assure, d'une part, le respect de la légalité de la peine et d'autre part, l'égalité de la peine ${ }^{71}$. L'autorité de la Cour s'exerce une fois de plus en cas d'évasion du condamné, car elle peut intervenir pour demander à l'État dans lequel le condamné se trouve, sa remise à l'État où il purgeait sa peine ou à un autre État désigné par la Cour ${ }^{72}$. En ce qui concerne la Cour, en l'absence d'États disponibles pour l'exécution, la peine d'emprisonnement est purgée dans un établissement pénitentiaire fourni par l'État hôte, à savoir les Pays-Bas ${ }^{73}$.

11 En vue de sa rééducation, l'accusé jouit de la faculté de demander une réduction de la peine, qui n'est possible que s'il a déjà purgé les deux tiers de la peine ou a déjà accompli 25 années d'emprisonnement, en cas de condamnation à perpétuité ${ }^{74}$. En matière de réduction de la peine, la règlementation détaillée souligne la protection statutaire $d u$ détenu ${ }^{75}$. Après avoir entendu le condamné, la Cour réexamine périodiquement sa situation et décide de la réduction, alors que l'État chargé de l'exécution n'est pas tenu de libérer la personne avant la fin de la peine prononcée par la C.P. ${ }^{76}$. En outre, après une première décision négative sur la réduction, la Chambre d'appel désigne trois juges chargés de réexaminer la question tous les trois ans et, s'ils trouvent les circonstances qui justifiaient la détention sensiblement modifiées, ils autorisent le condamné à demander un réexamen de la question ${ }^{77}$. La Cour décide donc favorablement lorsqu'elle observe un changement des circonstances qui avaient déterminé la peine ou lorsqu'elle constate un comportement coopératif spontané de l'accusé à l'égard des enquêtes, mais surtout à l'égard de l'exécution des mesures ordonnées en faveur des victimes ${ }^{78}$ : les juges doivent évaluer l'existence de toute action significative que le condamné a entrepris en faveur des victimes et quelle répercussion sur celles-ci peut avoir sur une libération anticipée ${ }^{79}$. Au travers de l'analyse de la réglementation sur la réduction de la peine, il faut noter l'effort entrepris pour équilibrer deux intérêts opposés, d'une part celui du condamné à faire valoir ce qui pourrait être défini comme un vrai droit à la réduction - un droit qui, toutefois, est conditionné par la vérification d'une série de conditions -, et d'autre part les intérêts des victimes à la réparation, à la protection et à la vérité. Le respect des droits de ces parties est démontré par la procédure nécessaire pour la décision portant sur la réduction de la peine. Il s'agit d'une procédure qui reproduit le procès, dans la mesure où elle se déroule au cours d'une audience tenue à titre exceptionnel, où le condamné peut se faire assister par un conseil et où toutes les autres parties impliquées, à savoir le Procureur, les États chargés de l'exécution, les victimes et leurs représentants légaux sont invitées à participer. Afin de décider sur la réduction, la Cour devra se tenir à d'autres critères, tels que la possibilité de réinsertion sociale, les conditions de santé physique ou mentale, l'âge du condamné, l'absence de risques pour la stabilité sociale qu'une libération anticipée pourrait causer, sans négliger la démonstration que la période de détention a produit sur l'accusé une volonté de désavouer le crime ${ }^{80}$.

12 Un problème épineux au sein de la justice pénale internationale est celui lié à la durée de la détention avant procès. Au-delà de l'indemnisation, parmi les formes de 
réparation reconnues il y a la réduction de la peine à purger en rapport avec le nombre d'années que l'accusé a déjà passé en détention provisoire dans le cadre de la même procédure et pour les mêmes faits. Il peut s'agir d'un nombre considérable d'années de détention subies par le justiciable avant procès, dont la durée varie en fonction de la durée totale de la procédure ${ }^{81}$. La période de la détention déjà purgée à titre provisoire est alors prise en compte au moment du calcul de la peine, mais cela ne doit pas être confondu avec la réduction de la peine, cette dernière concernant une peine que l'accusé a déjà commencé à purger en tant que condamnés ${ }^{82}$. Le problème de la détermination du quantum se pose donc aux juges. À cet égard, le Statut de Rome a règlementé et donné plus de précision au développement jurisprudentiel qui s'était produit en la matière au sein des T.P.I., qui ont souvent dû aborder des situations où la personne suspectée, avant de comparaitre pour la première fois devant les juges, avait déjà subi une détention au niveau national qui était en relation avec le crime poursuivi par la juridiction internationale ${ }^{83}$. Selon l'article 78(2), afin de calculer la détention finale, il faut d'abord définir le début de la période de la détention provisoire appliquée sous l'ordre de l'instance internationale. Toutefois, des problèmes de calcul se posent quand la détention provisoire n'a pas été subie sous l'ordre de la Cour. À cet égard, le Statut de Rome révèle sa fonction de garantie des droits de la défense, lorsqu'il spécifie que la détention avant procès à prendre en compte n'est pas uniquement celle qui dérive d'une décision de la Cour. Autrement dit, la Chambre, au moment où elle prononce une peine d'emprisonnement, doit, d'une part, considérer le temps que l'accusé a passé en détention "sur son ordre ", mais, d'autre part, "elle peut également en déduire toute autre période passée en détention à raison d'un comportement lié au crime " ${ }^{84}$. La condition imposée par le Statut est néanmoins que le comportement qui a justifié la détention provisoire soit lié au crime sur lequel la compétence de la Cour s'est étendue. Les interprétations données par la jurisprudence des T.P.I. à cet égard n'ont pas donné preuve d'uniformité en ce qui concerne le moment où il fallait calculer le début de la détention provisoire, surtout de la détention subie sur le territoire national, loin de La Haye. Dans certains cas, la jurisprudence a fait partir le calcul au moment où l'ordonnance est émise par la Chambre ou au moment où la requête est présentée par le Procureur aux autorités nationales ${ }^{85}$, alors que, dans d'autres cas, l'interprétation a fait coïncider le début du calcul avec la comparution volontaire de l'accusé devant le Tribunal ${ }^{86}$. En d'autres termes, au moment de la condamnation à une peine d'emprisonnement, le Statut de Rome reconnaît aux juges le pouvoir de déduire de la peine totale la détention déjà purgée avant la condamnation, même la détention échappant au contrôle de la Cour et passée devant les juridictions nationales pour des crimes liés aux crimes pour lesquels l'accusé a été condamné par la C.P.I. ${ }^{87}$. Il s'agit d'une extension et d'un automatisme se détachant de la volonté du condamné d'exercer ce droit et qui semble vouloir donner une forme de réparation non uniquement économique à la privation de la libertée ${ }^{88}$. Une détention provisoire très prolongée serait alors considérée comme tolérable par le fait qu'ensuite, au moment de la condamnation, l'accusé jouira toujours d'une réduction de peine.

\section{Conclusion}

13 La réflexion critique sur le régime de la peine en vigueur devant la C.P.I. révèle un arsenal rigoureux quant aux prévisions normatives applicables par rapport aux systèmes des T.P.I., expression d'un système statutaire qui répudie toute vengeance et 
qui souligne, en revanche, le rôle dissuasif de la peine. De failles sont néanmoins présentes, comme par exemple le fait que le Statut de Rome se prononce sur le type de sanction applicable mais il reste plus lacunaire et générique quant aux critères à adopter pour leur application. En outre, le manque de prévision d'une grille de peines maximales et minimales pour chaque crime risque de donner trop de pouvoir arbitraire aux juges. Un autre point de faiblesse est lié à la détention, en particulier à la détention provisoire. Le problème reste chronique, puisqu'il s'agit d'une pratique encore trop largement diffusée dans le milieu de la justice pénale internationale. Dans le contexte de la justice pénale internationale moderne, les deux perspectives se rencontrent, convergeant vers le but de favoriser la réconciliation et garantir l'ordre et la paix internationale, car la peine sortant de la justice internationale doit satisfaire l'opprobre et la stigmatisation de la part de la communauté internationale envers certaines conduites, neutraliser le coupable et prévenir toute conduite de la même espèce. À son début, la justice pénale internationale visait essentiellement au châtiment du coupable, qui pouvait être condamné à la peine de mort suite à un procès sommaire, dépourvu de tout respect des règles procédurales garantissant un procès équitable ${ }^{89}$. Après l'expérience des T.P.I., la C.P.I. exprime et souligne cette mixité d'approche, qui répudie toute idée de vengeance sociale et qui souligne plutôt la centralité du rôle dissuasif de la peine dans la prévention d'autres crimes si odieux ${ }^{90}$.

\section{NOTES}

1. I.C.C., Le Procureur v. Germain Katanga, Chambre d'appel, Decision on the review concerning reduction of sentence of Mr Germain Katanga, 13 novembre 2015, No. ICC-01/04/01/07.

2. Voy. le Rapport du Comité préparatoire pour la création d'une cour criminelle internationale, doc. off. NU A/CONF.183/2/Add.1 (14 avril 1998), pp.122-124. Certains États Parties, comme l'Arabie Saoudite et Singapour, proposaient la peine de mort, admise au sein de leur systèmes nationaux, alors que d'autres s'y opposaient; doc. off. NU A/CONF.183/C.1/WGP/L.13 (juillet 1998) ; R.-E. FIFE, "Penalties", in R.-S. LEE (ed.), The International Criminal Court: The Making of the Rome Statute, Issues, Negotiations, Results, Kluwer Law International, The Hague, 1999, pp. 319-344.

3. D'ailleurs, pareillement à ce que l'art. 22(3) établit à propos des crimes, le Statut de la Cour prévoit que ses normes n'affectent pas l'application dans les systèmes nationaux de peines autres que celles admises par la Cour, de même quand la compétence nationale est exercée sur les crimes de sa compétence. Plus spécifiquement, l'article 80 du Statut de Rome établit que «rien dans le présent chapitre n'affecte l'application par les États des peines que prévoit leur droit interne, ni l'application du droit des États qui ne prévoient pas les peines prévues dans le présent chapitre ». A cet égard, il faut reprocher la disparité existante entre le régime des sanctions de la C.P.I. et celui de certains États Parties, qui restent titulaires d'une compétence primaire : cela affecte le principe de légalité de la peine, selon que l'accusé soit poursuivi par la Cour ou par la juridiction nationale qui applique la peine capitale. Des problèmes analogues s'étaient posés avec les T.P.I. qui n'appliquaient pas la peine de mort, alors qu'elle était admise au niveau national.

4. $C f$. Convention pour la prévention et la répression du crime de génocide, adoptée par la Résolution 260 (III) A de l'Assemblée générale des Nations Unies, le 9 décembre 1948 ; Convention de Genève relative à la protection des personnes civiles en temps de guerre, 12 août 1949; 
Convention contre la torture et autres peines ou traitement cruels, inhumains ou dégradants, adoptée par la Résolution 39/46 de l'Assemblée générale des Nations Unies, le 10 décembre 1984.

5. A cet égard voir, W.-A. SCHABAS, "Perverse Effects of the Nulla Poena Principle: National Practice and the Ad Hoc Tribunals", 11(3), E.J.I.L. (2000), p. 521.

6. C.P.I., art. 77(2).

7. À cet égard, le Statut indique la restitution, l'indemnisation ou la réhabilitation. Dans ce cas, l'art. 75(1) établit que la Cour « peut » et non « doit » déterminer l'ampleur du dommage, de la perte et du préjudice à l'encontre des victimes et indiquer les principes à la base de la décision, C.P.I., art. 75(1)(3). Ce n'est que récemment que la Chambre d'appel a fixé les principes et la procédure à appliquer en matière de réparation, C.P.I, The Prosecutor v. Thomas Lubanga Dyilo, Judgment on the appeal against the "Decision establishing the principles and procedures to be applied to reparations" of 7 August 2012, 3 March 2015, ICC-01/04-01/06 AA2A3.

8. C.P.I., art. 78(1).

9. C.P.I., art. 76(1) du Statut de Rome.

10. À l'égard des distorsions dérivant du principe de légalité de la peine voy. W.-A. SCHABAS, ibid., p. 521 ; P. KOVACS, «Le prononcé de la peine », in H. ASCENSIO/E. DECAUX/A. PELLET, Droit international pénal, Editions A. Pedone, 2000, pp. 841-848.

11. Dans l'affaire Delalić, le T.P.I.Y. a considéré comme non contraignant le critère de la référence à la loi nationale applicable, par conséquent, la référence à la grille générale des peines d'emprisonnement offerte par le système national a été considérée plutôt comme une source d'inspiration par les T.P.I. ; M.-C. BASSIOUNI, Le fonti e il contenuto del diritto penale internazionale, Giuffrè, Milano, 1999, pp. 171-173.

12. Report of the International Law Commission on the Work of its Forty-Seventh Session, 2 May-21 July 1995, U.N. GAOR, $50^{\text {th }}$ Sess., Supp. N. 10, 68, U.N. Doc. A/50/10 (1995).

13. I.C.T.Y., The Prosecutor v. Drazen Erdemović, Trial Chamber, Sentencing Judgement, 29 November 1996, IT-96-22-T, §§ 35-40.

14. P. KOVACS, «Le prononcé de la peine », op. cit., p. 843.

15. C.P.I., art. 23, «Une personne qui a été condamnée par la Cour ne peut être punie que conformément aux dispositions du présent Statut ».

16. Voy. J.-R.-W.-D. JONES/S. POWLES, International Criminal Practice, Third edition, Oxford University Press, Oxford, 2003, pp. 404-405, où l'art. 24(1) du Statut du T.P.I.Y., qui utilise l'expression "shall have recourse to the general practice... » est comparé avec la règle 101(B) R.P.P., qui utilise l'expression "take into account». Dès le début de son activité, la C.D.I. a toujours souligné l'importance du principe de légalité de la peine et le Projet de Code des crimes contre la paix et la sécurité de l'humanité fut amendée afin de mieux garantir le respect du principe. L'A.G. avait établi que toute peine relative aux crimes internationaux devait tenir compte de la réglementation applicable au niveau international; voy. Report of the 1953 Committee on International Criminal Jurisdiction, UN Doc. A/2645, para. 118, voy. également voy. S. LAMB, "Nullum crimen, nulla pona sine lege in International Criminal Law », in A. CASSESE/P. GAETA/J.R.-W. JONES (dir.), The Rome Statute of the International Criminal Court: a Commentary, Oxford university Press, Oxford, 2002, p. 756 et s.

17. Voy. Report of the I.L.C. on the work of its $46^{\text {th }}$ Session ; voy. également Report of the ad hoc Committee on the Establishment of an International Criminal Court, UN GAOR, $50^{\text {th }}$ Sess., Supp. No. 22, A/50/22 (1995), at paras. 52 and 57.

18. À cet égard, le rapport du Comité préparatoire relatif à l'établissement de la Cour, soumis à la Conférence diplomatique de Rome en 1998, avait confirmé les divergences profondes existant entre les traditions juridiques des différents pays. Ladite observation faisait craindre que la procédure de la C.P.I. n'aurait pas pu respecter le principe de légalité de la peine, United Nations Diplomatic Conference of Plenipotentiaries on the Establishment of an International Criminal Court-Report 
of the Preparatory Committee on the Establishment of an International Criminal Court, addendum A/ CONF.183/2/Add.1, 14 April 1998.

19. Ladite difficulté avait été confirmée en 1996 par la Commission au cours des travaux pour la rédaction du Code des crimes contre la paix et la sécurité de l'humanité ; voy. Draft Code of Crimes against the Peace and Security of Mankind, Report of the ILC on the work of its $48^{\text {th }}$ Sess., 6 May-26 July 1996, UN Doc. A/51/10, at 29-30 ; Report of the International Law Commission on the Work of its $47^{\text {th }}$ Sess., UN Doc. A/50/10, at 183.

20. Voy. la décision de la Commission Préparatoire en 11-21 février 1997, Annexe II, UN Doc. A/ AC.249/1997/L.5, at 18.

21. Voy. UN Doc. A/CONF. 183/C.1/L.65/Rev.1, at 2 ; sur ce point il faut rappeler la proposition avancée par la délégation mexicaine à l'occasion de la Conférence diplomatique de Rome, qui demandait au Groupe de travail sur les peines d'introduire le principe de légalité de la peine, UN Doc. A/CONF.183/C.1/WGP/L.4.

22. C.P.I., règles 145 à 148 R.P.P.

23. Voy. le Chapitre 12 du R.P.P en matière d'exécution.

24. À cet égard voy. les nombreuses prononciations des T.P.I. concernant la fonction de la peine : T.P.I.Y., Le Procureur v. Drazen Erdemović, Chambre de première instance I, Jugement portant condamnation, cit., § 59-64; T.P.I.R., Le Procureur v. Jean Kambanda, Chambre de première instance, Jugement, 4 septembre 1998, ICTR-97-23-S, § 28, qui confirment la centralité de la fonction de dissuasion, rétribution, stigmatisation publique et réconciliation nationale attribuée à la peine.

25. A.-M. LA ROSA, Juridictions pénales internationales, PUF, Paris, 2003, p. 160.

26. Voy. C. BECCARIA, Dei delitti e delle pene, où l'Auteur souligne la fonction démonstrative et dissuasive de la peine, qui doit être proportionnée et doit éviter toute forme de cruauté envers le condamné ; voy. également J. BENTHAM, Théorie des peines et des récompenses, 3e éd., Bossange, Paris, 1825, p. 16 et s.

27. Voy. également les dispositions analogues des T.P.I., l'art. 24(2) du T.P.I.Y et l'art. 23(2) du T.P.I.R.

28. C.P.I., art. 76(1) du Statut de Rome.

29. C.P.I., règle 145 R.P.P., T.P.I, art. 101(B)(i)(ii).

30. Ibid., règle 145(1)(a)(b) R.P.P. ; la jurisprudence des T.P.I. a confirmé ce critère, voy. T.P.I.Y., Le Procureur v. Erdemovic, Jugement portant condamnation I, cit., $\S \S 105$ et 110 ; T.P.I.Y., Le Procureur v. Tadić, Jugement portant condamnation, cit., par. 62 ; T.P.I.R., Le Procureur v. Akayesu, Décision relative à la condamnation, 2 octobre 1998, ICTR-96-4, § 33.

31. C.P.I., règle $142(1)(c)(2)(b)$ R.P.P.

32. Voy. T.P.I.Y., Le Procureur v. Celebici, Chambre d'appel, Jugement, cit., §§ 755-759; à cet égard A.-M. LA ROSA, Juridictions pénales internationales, op. cit., pp. 191-192.

33. C.P.I., règle 145(1)(b) R.P.P.

34. Rapport du Groupe de travail sur les peines, doc. off. NU A/CONF.183/C.1/WGP/L.14 (4 juillet 1998).

35. C.P.I., règle 145(2)(b) R.P.P.; voy. particulièrement les précédents jurisprudentiels : Le Procureur v. Akayesu, Décision relative à la condamnation, cit., § 36 ; T.P.I.Y., Le Procureur v. Celebici, Jugement, cit., $\S \$ 1217,1223,1235,1243,1244,1251$, 1268, 1269, et 1272 ; T.P.I.R., Le Procureur v. Musema, Jugement, cit., §§1003-1004 ; T.P.I.R., Le Procureur v. Rutaganda, Jugement et sentence, 6 décembre 1999, ICTR-96-3, §§ 468-470.

36. Voy. T.P.I.R., Le Procureur v. Kambanda, Jugement portant condamnation, cit., § 33 ; T.P.I.R., Le Procureur v. Ruggiu, Jugement, cit., § 48.

37. C.P.I., règle 145(2)(a) R.P.P.; voy. également la jurisprudence des T.P.I., en particulier: T.P.I.Y., Le Procureur v. Erdemovic, Jugement portant condamnation, cit., $\S \S 44$ et 55 et sentence, cit., $\S \S 16$ et 21 ; T.P.I.R., Le Procureur v. Musema, Jugement, cit., §§ 1005 et 1007 ; T.P.I.R., Le 
Procureur v. Ruggiu, Jugement, cit., §§ 56-58 et §§ 69-72 ; T.P.I.Y., Le Procureur v. Celebici, Jugement, cit., § 1279 .

38. C.P.I., Situation en République Démocratique du Congo. Le Procureur v. Germain Katanga, Chambre de première instance II, Décision relative à la peine (article 76 du Statut), 26 mai 2014, ICC-01/04-01/07, § 144.

39. C.P.I., art. 27.

40. Procès des grands criminels de guerre devant le Tribunal militaire international, Nuremberg, 14 novembre 1945-1 ${ }^{\text {er }}$ octobre 1946, Jugement, tome I, pp. 235-236.

41. T.P.I.Y., Le Procureur v. Erdemovic, Jugement portant condamnation, cit., § 47.

42. C.P.I., art. 33. A cet égard cf. la jurisprudence des T.P.I. : I.C.T.Y., The Prosecutor v. Sikirica, Sentencing Judgement, 13 November 2001, IT-95-8, § 192.

43. En particulier, il s'agissait de considérer dans le calcul les conditions de détention subies par l'accusé en R.D.C., voy. C.P.I., Situation en République Démocratique du Congo. Le Procureur v. Germain Katanga, Décision relative à la peine (article 76 du Statut), 26 mai 2014, ICC-01/04-01/07, §§ 130 à 133 et 136 à 138.

44. Par exemple les articles 103(1)(2) et 106. Sur ce point voy. en particulier, M.-C. BASSIOUNI, Introduction au droit pénal international, Bruylant, Bruxelles, 2002, p. 275.

45. C.P.I., art, 77. Sur le sujet voy. M.-C. BASSIOUNI, Le fonti e il contenuto del diritto penale internazionale, op. cit., p. 173 ; en matière de peines et d'execution des peines, voy. surtout D. SCALIA, Chronique de droit international pénal, R.D.I.P. (2014/3) vol. 85.

46. Le $1^{\mathrm{er}}$ décembre 2014, la Chambre d'appel de la Cour pénale internationale a confirmé, à la majorité des juges, la décision déclarant la culpabilité de M. Lubanga ainsi que celle le condamnant à une peine de 14 ans d'emprisonnement. Le 14 mars 2012 l'accusé avait été déclaré coupable et le 10 juillet 2012 il avait été condamné à une peine totale de 14 ans d'emprisonnement, de laquelle serait déduit le temps qu'il avait passé en détention sous ordre de la C.P.I. ; la peine, selon la Chambre, reflétait donc la culpabilité de l'accusé pour les crimes pour lesquels il a été déclaré coupable ; C.P.I., Le Procureur v. Thomas Lubanga Dyilo, Chambre d'appel, Judgment on the appeals of the Prosecutor and Mr Thomas Lubanga Dyilo against the "Decision on Sentence pursuant to Article 76 of the Statute", 1 janvier 2014, ICC-01/04-01/06-3122 et [même affaire] Chambre d'appel, Jugement rendu en application de l'article 74 du Statut, 14 mars 2012.

47. Voy. le Projet de Statut de 1994 de la C.D.I., reproduit in Annuaire C.D.I. 1994, vol. II, 2e partie, art. 47(1)(a). Pendant les négociations au sein de la Conférence de Rome, plusieurs États s'étaient opposés aussi bien à l'emprisonnement à vie qu'à celui de très longue durée, considérés d'une sévérité excessive.

48. C.P.I., art. 77.

49. P. KOVACS, «Le prononcé de la peine », op. cit., p. 848.

50. T.P.I.Y., Le Procureur v. Tadić, Chambre d'appel, Arrêt relatif à la sentence, 26 janvier 2000, cit. et [même affaire] Jugement relatif à la sentence, 11 novembre 1999, avec l'opinion individuelle du juge Robinson.

51. T.P.I.R., Le Procureur v. Akayesu, Jugement, cit., § 8.

52. À cet égard, il faut mette en exergue une nouvelle tendance de la pénologie moderne qui considère plus le coupable que le crime, comme démontré par le T.P.I.Y. dans l'affaire Tadić, qui s'est attardé beaucoup sur la personnalité, l'âge, la classe sociale, l'indigence, le casier juridique vierge, l'effet de la peine sur la vie familiale du condamné; I.C.T.Y., The Prosecutor v. Tadić, Chamber II, Sentencing Judgement,14 July 1997, cit.

53. Voy. la comparaison entre l'art. 77(1)(b) et l'art. 78(1). C.P.I., art. 78(1) : “Lorsqu'elle fixe la peine, la Cour tient compte, conformément au Règlement de procédure et de preuve, de considérations telles que la gravité du crime et la situation personnelle du condamné".

54. Ibid., règle 145(3) R.P.P. 
55. Dans cette procédure, il faut cependant distinguer entre la ou les circonstances aggravantes et la situation personnelle du condamné ou la gravité extrême de la conduite.

56. C.P.I., art. 77(2)(a)(b) et règle 146 R.P.P.

57. Cependant, l'amende et la confiscation ne représentent pas une forme directe de réparation puisqu'elles ne correspondent pas à l'indemnisation et à la réparation proprement dites, étant destinées au Fond crée par les États Parties au profit des victimes et non directement aux victimes, Ibid., art. 79 et 75. Voy. M.-C. BASSIOUNI, Le fonti e il contenuto del diritto penale internazionale, op. cit., pp. 173-180 ; M.-C. BASSIOUNI Introduction au droit pénal international, op. cit. ,p. 277-283.

58. C.P.I., règle 97(2) R.P.P.

59. Ibid., art. 109(1) et règle 147 R.P.P.

60. Ibid., art. 79(2).

61. Ibid., règle 148 R.P.P.

62. Ibid., règles 217 à 222 R.P.P.

63. Sur cet aspect voy, H. ABTAHI, "Execution de la peine", in H. ASCENSIO/E. DECAUX/A. PELLET, Droit international pénal, Deuxième édition révisée, Editions A. Pedone, 2012, Paris, pp. 989-992 ; E. LAMBERT-ABDELGAWAD, « L'emprisonnement des personnes condamnées par les juridictions pénales internationales : les conditions relatives à l'aménagement de la peine ", R.C.S. (2003) $n^{\circ} 1$, pp. 162-171.

64. C.P.I., art. 103(3)(a).

65. Ibid., art. 103 ; voy. également surtout la pratique du T.P.I.Y. in Annuaire du T.P.I.Y. (1994), p. 161 et s., 1995, p. 329 et s., 1996 p. 226 et s.

66. Voy. P. KOVACS, «Le prononcé de la peine», in H. ASCENSIO/E. DECAUX/A. PELLET, Droit international pénal, op. cit., p. 849-854.

67. C.P.I., art. 106.

68. Ibid., art. 110.

69. Ibid., art. 105(1).

70. Ibid., art. 106(3).

71. En particulier, les normes internationalement reconnues sont: les Principes fondamentaux relatifs au traitement des détenus adoptés par l'Assemblée générale des Nations Unies dans sa résolution 45/111, le 14 décembre 1990 et l'Ensemble de principes pour la protection de toutes les personnes soumises à une forme de détention quelconque ou d'emprisonnement, adopté par l'Assemblée générale des Nations Unies dans sa résolution 43/173, le 9 décembre 1988.

72. C.P.I., art. 111.

73. Ibid., art. 103(4).

74. Ibid., art. 110(2).

75. M.-C. BASSIOUNI, Introduction au droit pénal international, op. cit., pp. 276-277.

76. C.P.I., art. $110(1)(2)(3)$.

77. Ibid., règle 224(3) R.P.P.

78. Ibid., art. 110(4).

79. Ibid., règle 223(d) R.P.P.

80. Ibid., règle 223(a)(b)(c)(e) R.P.P.

81. Dans l'affaire Lubanga, la Chambre a condamné l'accusé à 14 années d'emprisonnement, déduisant de la peine le temps que l'accusé a passé en détention à partir de la date de son arrestation, le 16 mars 2006, jusqu'à celle de la sentence sur la peine, sans considérer la détention passé dans son pays, en l'absence de preuves suffisantes à établir que Lubanga avait été détenu en R.D.C. à raison d'un comportement lié aux crimes dont il a été reconnu coupable par la Cour.

82. C.P.I., art. 78(2).

83. Dans l'affaire Kaing Guek Eav, alias Duch, devant les C.E.T.C., pour le calcul de la peine, la Chambre d'appel a dû tenir compte du nombre considérable d'années de détention, du 10 mai 
1999 au 30 juillet 2007, purgée devant les autorités nationales avant que la condamnation soit prononcée par les C.E.T.C., voy. E.C.C.C., Supreme Court Chamber, Case 001 Appeal Judgement, 3 February 2012, 011/18-07-2007-ECCC/SC, §§ 400-405.

84. Ibid., art. 78(2).

85. T.P.I.Y., le Procureur v Kupreskic, cit., Chambre d'appel, Jugement, 14 janvier 2000, dispositif, cit.; T.P.I.Y., Le Procureur v. Celebici, Jugement, cit., §§ 1287-1289 ; T.P.I.R., Le Procureur v. Ruggiu, Jugement, verdict, cit.

86. T.P.I.Y., Le Procureurv $v$ Blaskic, Jugement, 3 mars 2000, IT-95-14, cit., §41.

87. La question du crédit pour la détention provisoire était déjà prévue dans l'art. 77 du Projet de Statut du Comité préparatoire pour la création d'une cour criminelle internationale.

88. A.-M. LA ROSA, Juridictions pénales internationales, op. cit., pp. 201-202.

89. Cf. l'affaire Eichmann devant la Cour suprême d'Israël, in I.L.R, vol. XXXVI (1968), p. 341 ; T.P.I.Y., Premier Rapport annuel à l'Assemblée générale et au Conseil de sécurité, doc. off. NU AG A/49/342, doc. off. NU CS/1994/1007 (29 août 1994), par. 11 à 16; voy. T.P.I.R., Le Procureur v. Ruggiu, Jugement, 1 juin 2000, ICTR-97-32-I, § 33 ; T.P.I.R., Le Procureur v. Musema, Jugement, 27 janvier 2000, ICTR-96-13, §986 ; I.C.T.Y., Le Procureur v. Kupreskic, Jugement, 14 janvier 2000, IT-95-16, § 848 ; I.C.T.Y., Le Procureur v. Erdemovic, Jugement portant condamnation, 29 novembre 1996, cit., § 66 .

90. Voy, le Préambule au Statut de la C.P.I. «Déterminés à mettre un terme à l'impunité des auteurs de ces crimes et à concourir ainsi à la prévention de nouveaux crimes "; voy. également A. SOTTILE, "Le problème de la création d'une cour pénale internationale permanente", Revue de droit international (1951), vol. XXIX, p. 163.

\section{RÉSUMÉS}

Le principe de légalité de la peine demeure incertain en droit pénal internationale et la règlementation de la cour pénale internationale confirme cette tendance. Il s'agit d'une incertitude qui, toutefois, exige que la sanction se conforme aux critères de proportionnalité et d'individualisation, deux critères que la peine doit donc respecter.

Legality principle of penalty is uncertain in international criminal law and the International Criminal Court rules points out such a character. It is a character that, moreover, lets that sanction respects proportionality and individualizations in penalty

\section{INDEX}

Mots-clés : cour pénale internationale, juridictions pénales internationales, peine, légalité, proportionnalité, individualisation, crime, exécution, accusé.

Keywords : international criminal court, international criminal jurisdictions, penalty, legality, proportionality, individualisation, crime, execution, accused. 
AUTEUR

MARIA STEFANIA CATALETA

Maria Stefania Cataleta est avocate, docteur en droit et sciences politiques 